\title{
Hybridization Using a New Male-sterile Germplasm as the Female Parent in Chinese Jujube
}

\author{
Jiu-rui Wang ${ }^{1}$, Xiu-mei Cui ${ }^{1}$, Li Dai ${ }^{2}$, Ping Liu' ${ }^{2}$, Jin Zhao ${ }^{3}$, and Meng-jun Liu² \\ ${ }^{1}$ College of Forestry, Agricultural University of Hebei, Baoding 071001, P.R. China \\ ${ }^{2}$ Research Center of Chinese Jujube, College of Horticulture, Agricultural University of Hebei, No. 289, Lingyusi Road, Baoding 071001, P.R. China \\ ${ }^{3}$ College of Life Science, Agricultural University of Hebei, Baoding 071001, P.R. China
}

\begin{abstract}
This study identified a new male-sterile germplasm of Chinese jujube, named male-sterile No. 2 (JMS2), and achieved controlled hybridization using that germplasm as the female parent. The anthers of JMS2 before flower bud opening became shrunken, dingy yellow and much smaller than normal ones, and they changed to brown after anthesis. No pollen was observed in anthers of JMS2 and its male-sterile trait remained stable over different years. A total of 1,642 fruits were obtained from ten intra- and interspecific cross combinations via controlled hybridization from 2008 to 2012 using JMS2 as the female parent. Of those, $27.3 \%$ produced seeds, on average (0-72.6\%). The rate of fruit with seed (RFS) was significantly different between cross combinations or years. Compared to other cross combinations, the RFS in the combination of JMS2 $\times$ 'Xingguang' (a Chinese jujube cultivar with high resistance to jujube witches' broom disease) and JMS2 $\times$ 'Xing16' (a sour jujube genotype) remained high in different years and reached means of 48.7 and 58.1\%, respectively. Finally, 150 plantlets were regenerated from immature embryos, and 51 of them were randomly selected and identified to be authentic hybrids using amplified fragment length polymorphism (AFLP) markers. This is the first report of hybrids obtained from a cross between Chinese jujube and sour jujube.
\end{abstract}

Additional key words: embryo culture, hybrid, interspecific cross, pollen, sour jujube

\section{Introduction}

Chinese jujube (Ziziphus jujuba Mill.) is one of the most important native fruit trees of China with growing area of 2 million ha and annual production of over 5 million tons. It has been introduced into more than 30 countries directly or indirectly from China (Liu, 2006; Lyrene, 1983; Yao, 2013). The fruit of Chinese jujube is becoming more popular as its nutritional and medical values have been demonstrated. Currently, growers and consumers expressed interest in new cultivars releasing because of the rapid expansion of the market. Yet, almost all the Chinese jujube cultivars are obtained by selection breeding through observation on the variants within traditional cultivars or investigation of the seedlings derived from natural pollinations
(Liu, 2006; Liu and Wang, 2009). Only one tetraploid cultivar 'Chenguang' was derived from colchicine-treated diploid cultivar 'Linyilizao' (Liu et al., 2012).

Cross breeding is the basic breeding approach in fruit breeding. Yet, no cultivar of Chinese jujube bred via cross has been released up to now. The cross breeding of Chinese jujube faces several severe obstacles, such as severely difficult emasculation, very low fruit set rate, and heavy embryo abortion. Embryo abortion takes place in more than half of the Chinese jujube cultivars, which causes either no seed or shriveled kernels in fruits. Especially, most excellent cultivars that might serve as parents have serious embryo abortion (Liu, 2006). In addition, the flower buds of Chinese jujube are very small ( $3 \mathrm{~mm}$ in diameter) and it is almost impossible to emasculate the flowers

\footnotetext{
*Corresponding author:1mj1234567@aliyun.com

※ Received 10 October 2014; Revised 19 January 2015; Accepted 29 January 2015. This work was supported by the National Science and Technology Support Program (2013BAD14B03) and the National Natural Science Foundation of China (30471194). Thanks are due to Prof. Kejiu Du for his kind assistance in manuscript revision.

(C) 2015 Korean Society for Horticultural Science
} 
without causing serious damage. Up to now, only seedlings obtained under natural pollination were reported in Chinese jujube (Lu et al., 2005). Except for manual emasculation, the prevention of self-pollination in hybridization could be achieved by using female parent of self-incompatibility or male sterility. Chinese jujube has been reported to have gametophytic self-incompatibility (Asatryan and Tel-Zur, 2013; Zhang et al., 2003). However, self-incompatibility genotype is unclear in Chinese jujube. Furthermore, many cultivars could produce fruits and seeds after self-pollination (Yan et al., 2009, 2010). Male sterile germplasm is usually seldom in natural germplasm. The authors screened 219 cultivars of Chinese jujube and found only one typical male sterile genotype named JMS1 (Liu, 2006; Wang et al., 2007). However, it has not been successfully used in the practical cross breeding because of its low embryo fertility. Thus, new male-sterile germplasms with good embryo fertility are highly expected.

The aim of the present study was to validate a new male-sterile germplasm of Chinese jujube and to achieve intra- and interspecific crosses using the germplasm as a female parent. Sour jujube (Z. acidojujuba Cheng et Liu) is widely distributed in northern China and mainly used as traditional Chinese medicine and the rootstock of Chinese jujube. To our knowledge, this is the first report on distant hybridization between Chinese jujube and sour jujube.

\section{Materials and Methods}

\section{Plant Material}

Nine Chinese jujube cultivars including a suspected malesterile germplasm discovered by chance in 2005 and four sour jujube genotypes grown at jujube experimental station of agricultural university of Hebei located in Xianxian County, Hebei, China were used in this study. The new male-sterile germplasm was named Chinese jujube male sterile No. 2 (JMS2), which had excellent table quality, late ripening fruit as well as high and stable yield.

\section{Identification of a Male-sterile Germplasm}

In order to validate the male-sterile trait of JMS2, pollen number per anther and anther morphology and structure of the germplasm and some male-fertile cultivars were investigated. Pollen number per anther was tested by using a hemocytometer (Zhang, 2001). Thirty anthers for each cultivar were sampled from the flower buds at yellow color stage and repeated three times, which were separately put into small empty bottles. Two milliliters of $1 \%$ sodium hexametaphosphate solution were added into each bottle after the anthers became dried completely and cracked. The pollen liquid was checked on a hemocytometer under a microscope, and the pollen number per anther was calculated. The anther samples for observation of anatomical structures in 2005 and 2006 were collected and immediately fixed in FAA fixative consisting of $38 \%(\mathrm{v} / \mathrm{v})$ formaldehyde, glacial acetic acid, and $70 \%(\mathrm{v} / \mathrm{v})$ ethanol in a ratio of 5:5:90. The procedure of paraffin section and microscopic observation were conducted as described by Li (1996).

\section{Controlled Hybridization and Culture of Hybrid Embryo}

JMS2 was used as a female parent in intra- and interspecific crosses. Six Chinese jujube cultivars and four sour jujube genotypes were chosen as male parents (Table 1). 'Xingguang' is a Chinese jujube cultivar with high resistance to jujube witches' broom disease (Liu et al., 2006) and 'Xing16' is a sour jujube genotype with big fruits. Controlled pollination between male-sterile and male-fertile plants was carried out from 2008 to 2012. To exclude unwanted pollinators, two parent trees adjacently grown in the field were covered together with a white nylon net $(4 \mathrm{~m} \times 6 \mathrm{~m} \times 3 \mathrm{~m}) 2$ to 3 days before flowering. A honeybee hive was placed into the net for pollination during flowering season.

The fruits were harvested at the end of August before ripening and kept at $4^{\circ} \mathrm{C}$ for 40 days. After the pulps were removed using a knife, the stones were cleaned in water and sterilized as follows: $75 \%$ ethanol for $2 \mathrm{~min}$, twice rinses in sterilized distilled water, $0.1 \% \mathrm{HgCl}_{2}$ for $8 \mathrm{~min}$, and three times of rinses in sterilized distilled water. The sterilized stones were smashed and the immature seeds inside were taken out. Then, the seed coats were stripped away and the embryos at late cotyledonal stage were placed onto Murashige and Skoog (MS) medium supplemented with $30 \mathrm{~g} \cdot \mathrm{L}^{-1}$ sugar and $3.3 \mathrm{~g} \cdot \mathrm{L}^{-1}$ agar (Wang et al., 2009). The regenerated plantlets from embryos were subcultured by shoot tip and stem in the MS medium supplemented with $30 \mathrm{~g} \cdot \mathrm{L}^{-1}$ sugar, $3.3 \mathrm{~g} \cdot \mathrm{L}^{-1}$ agar, $1.0 \mathrm{mg} \cdot \mathrm{L}^{-1}$ 6-benzylaminopurine and $0.5 \mathrm{mg} \cdot \mathrm{L}^{-1}$ indole-3-butyric acid (Wang et al., 2003).

\section{Hybridity Assessment with Amplified Fragment Length Polymorphism (AFLP) Markers}

Genomic DNA from the parents and progenies randomly selected, including 10 of 41 progenies in JMS2 $\times$ 'Xingguang' and 41 of 105 progenies in JMS2 $\times$ 'Xing16', was analyzed by AFLP markers. DNA extraction from young leaves of subcultured plantlets and AFLP fingerprinting were accomplished using the reported protocol with some modifications (Bai et al., 2009). Genomic DNA (400 ng) was digested in a $20-\mu \mathrm{L}$ volume including 3 units of $M s e I$ and EcoRI 
Table 1. Summary of controlled hybridization in Chinese jujube and sour jujube.

\begin{tabular}{|c|c|c|c|c|}
\hline Parents & Year & No. of fruits & No. of fruits with seeds & Rate of fruit with seed (\%) \\
\hline \multicolumn{5}{|l|}{ Intraspecific hybridization } \\
\hline \multirow[t]{4}{*}{ JMS2 × 'Wuhefeng' } & 2008 & 80 & 0 & 0 \\
\hline & 2009 & 7 & 0 & 0 \\
\hline & 2011 & 0 & 0 & - \\
\hline & 2012 & 0 & 0 & - \\
\hline \multirow[t]{2}{*}{ JMS2 × 'Longzao' } & 2008 & 0 & 0 & \\
\hline & 2011 & 1 & 0 & 0 \\
\hline JMS2 × 'Cangxiandasuanzao' & 2009 & 18 & 3 & 16.7 \\
\hline JMS2 × 'Changhong' & 2009 & 0 & 0 & - \\
\hline JMS2 × 'Lajiaozao' & 2009 & 0 & 0 & - \\
\hline \multirow[t]{3}{*}{ JMS2 × 'Xingguang' } & 2009 & 197 & 34 & 17.3 \\
\hline & 2011 & 15 & 9 & $60.0^{*}$ \\
\hline & 2012 & 83 & 57 & $68.7^{*}$ \\
\hline Subtotal intraspecific hybridization & & 401 & 103 & 25.7 \\
\hline \multicolumn{5}{|l|}{ Interspecific hybridization } \\
\hline \multirow[t]{3}{*}{ JMS2 × 'Xing16' } & 2009 & 175 & 127 & 72.6 \\
\hline & 2011 & 19 & 6 & $31.6^{*}$ \\
\hline & 2012 & 282 & 198 & 70.2 \\
\hline JMS2 × 'Xing6' & 2009 & 176 & 8 & 4.5 \\
\hline JMS2 × 'Xingtai0616' & 2009 & 324 & 2 & 0.6 \\
\hline \multirow[t]{2}{*}{ JMS2 × 'Xing27' } & 2008 & 101 & 1 & 0.9 \\
\hline & 2009 & 164 & 3 & 1.8 \\
\hline Subtotal interspecific hybridization & & 1,241 & 345 & 27.8 \\
\hline Total & & 1,642 & 448 & 27.3 \\
\hline
\end{tabular}

*Significantly different from the initial year in a cross combination by $t$ test, $p=0.05$.

each at $37^{\circ} \mathrm{C}$ for $5 \mathrm{~h}$. Adaptors were ligated with T4 DNA ligase at $16^{\circ} \mathrm{C}$ for $14 \mathrm{~h}$. Pre-selective reactions were carried out in a $20-\mu \mathrm{L}$ volume containing $5 \mu \mathrm{l}$ of ligation product, 0.5 units of Taq DNA polymerase and 50 ng of each primer. Selective amplifications were carried out in a $20-\mu \mathrm{L}$ volume containing $5 \mu \mathrm{L}$ of template DNA, 0.6 units of Taq DNA polymerase, and $50 \mathrm{ng}$ of each primer with three selective nucleotides.

To verify hybrids, we relied only on those AFLP markers, which were expressed strongly in the male parent and were not visible in the female parent. Transmission to a progeny of at least $50 \%$ of the male-specific markers was considered evidence that the progeny was a real hybrid (Pooler et al., 2002). If fewer than half of paternal markers were observed in a progeny, the hypothesis that the progeny was a real hybrid must be tested by calculating the probability of obtaining such a small number of markers in true hybrid of the putative parent through random assortment among the loci. If the probability was less than $0.05 \%$, then the hypothesis that the progeny was a real hybrid was rejected (Whittemore and Townsend, 2007).

\section{Results}

\section{Confirmation of Male Sterility in JMS2 via Morphological and} Anatomical Observation

Obvious morphological differences of anthers were observed between the suspected male-sterile and male-fertile germplasms at the yellow color stage of flower buds. Anthers of JMS2 were small, shrunken and dingy yellow (Fig. 1A) 
and became brown at the petal-anther separating stage. In contrast, anthers of male-fertile cultivars were plumpness, fresh yellow (Fig. 1B) and kept fresh yellow when petals were separating from anthers. While no normal pollen or

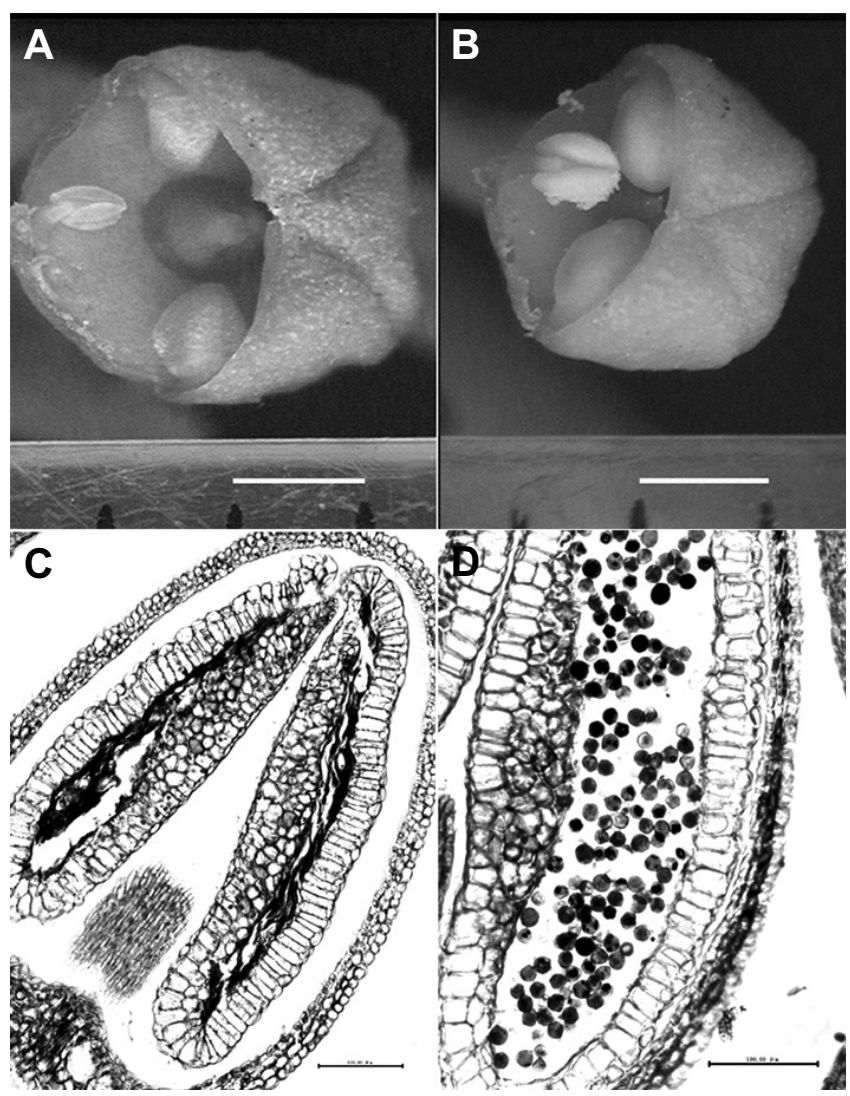

Fig. 1. Morphology and structure of anther in JMS2 (A and C) and 'Yueguang' (B and D). Bar = $1 \mathrm{~mm}$ (A and B). Bar $=100 \mu \mathrm{m}(\mathrm{C}$ and $\mathrm{D})$.

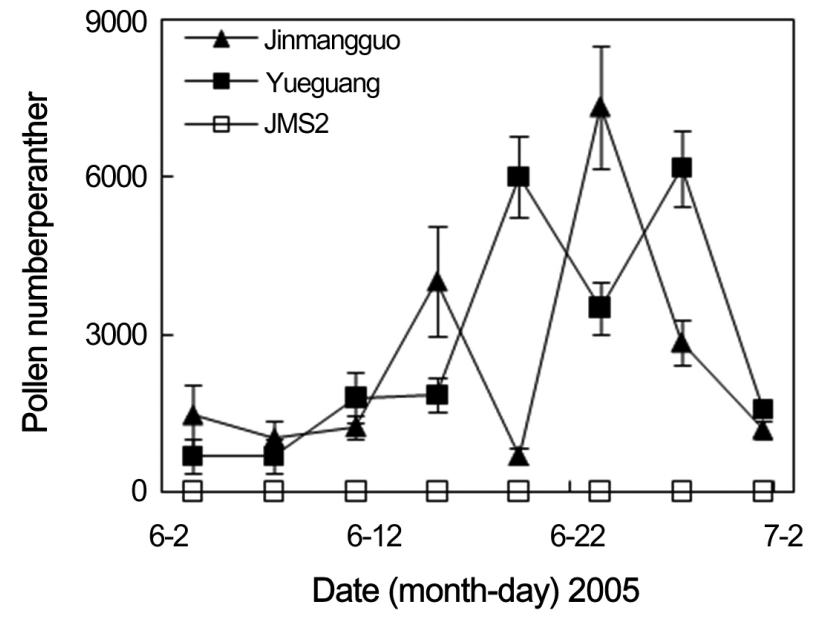

only pollen vestiges remained in JMS2 (Fig. 1C), the anthers of male-fertile cultivars had full of pollens (Fig. 1D). The anatomical structure of the anther in JMS2 was similar to that of JMS1 (Wang et al., 2007).

\section{Stability of Mail-sterile Trait in JMS2}

To confirm the stability of mail-sterile trait of JMS2, the changes of pollen production during flowering season were investigated in JMS2, 'Jinmangguo', 'Yueguang', and 'Lajiaozao' (Fig. 2). Pollen production changed a lot during flowering season in 'Jinmangguo', 'Yueguang', and 'Lajiaozao'. The pollen number per anther of 'Jinmangguo', 'Yueguang', and 'Lajiaozao' reached the highest around the middle of June, the peak days of flower opening, in 2005 and 2006. Both the highest pollen number per anther and the peak of flower opening in 'Jinmangguo' and 'Lajiaozao' appeared at the beginning of June in 2007 (data not shown). These results indicated that the highest pollen production usually appeared at the peak time of flower opening in Chinese jujube. However, no pollen appeared in the anther of JMS2 during the whole flowering season in different years. Its male-sterile trait always kept stable.

\section{Controlled Hybridization Using the New Male-sterile Germplasm as Female Parent}

Except for JMS2 × 'Changhong' and JMS2 × 'Lajiaozao', other eight cross combinations produced fruits and a total of 1,642 fruits had been obtained from 2008 to 2012 (Table 1). However, most of the fruits from both intraspecific and interspecific hybridization did not contain seeds (embryos). Only 448 of the 1,642 fruits had seeds (embryos), with a rate of $27.3 \%$. The rate of fruit with seed (RFS) was

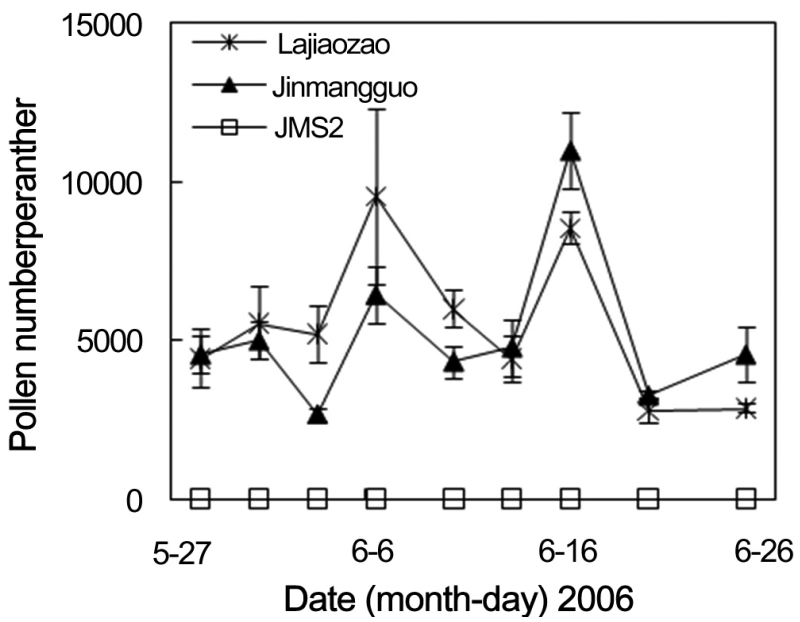

Fig. 2. Changes of pollen number per anther of different Chinese jujube germplasms during the flowering season. 
25.7 and $27.8 \%$ respectively in intraspecific and interspecific hybridization and the difference was not significant ( $t$ test, $p=0.05$ ). RFS ranged from 0 to $72.6 \%$ and varied among cross combinations and years. For instance, the RFS in JMS2 $\times$ 'Xing16' was significantly higher than other cross combinations in 2009 ( $t$ test, $p=0.05$ ). Yet there was no significant difference of RFS between JMS2 $\times$ 'Xing16' and JMS2 $\times$ 'Xingguang' in 2012 ( $t$ test, $p=0.05$ ). Compared with other cross combinations, the RFS in JMS $2 \times$ 'Xingguang' and JMS2 $\times$ 'Xing16' kept high level and reached a mean of 48.7 and $58.1 \%$, respectively.

\section{Hybridity Identification of Progenies}

Although eight of the ten cross combinations produced fruits, plantlet regeneration from immature embryos at late cotyledonal stage was only successful in four cross combinations (Table 2). Finally, 150 regenerated plantlets were obtained, including 105 from JMS2 $\times$ 'Xing16', 41 from JMS2 $\times$ 'Xingguang', 3 from JMS2 $\times$ 'Xing6', and one from JMS2 $\times$ 'Cangxiandasuanzao'. Six AFLP markers obtained from three primer pairs were used to identify the hybrids in JMS2 $\times$ 'Xing 16'. For example, one male-specific marker from the primer pair ATA/CAT was present only in 'Xing16' and some of the progenies (Fig. 3). Finally, at least 50\% (ranged from 3 to 5) male-specific markers were observed in every one of the 27 putative progenies. However, only one or two paternal markers appeared in every one of other 14 putative progenies. In contrast, four markers from the three primer pairs were used to verify hybrids in JMS2 $\times$ 'Xingguang'. The number of male-specific markers observed in every one of the seven putative progenies ranged from 2 to 4 . Every one of other three putative progenies only showed one male-specific marker. Thus, any offsprings had at least one of the six paternal markers in JMS2 × 'Xing16' or one of the four paternal markers in JMS2 × 'Xingguang'. The probability of any offspring inheriting one of the six paternal markers in JMS2 $\times$ 'Xing16' or one of the four paternal markers in JMS2 $\times$ 'Xingguang' was $p=0.09$ and 0.25 , respectively. Thus, the hypothesis that this plant was a hybrid should be acceptable. Finally, all progenies including those inheriting less than $50 \%$ of the male-specific markers were considered as authentic hybrids.

\section{Discussion}

The fruit set rate of Chinese jujube is very low and usually less than $2 \%$ of the flowers can develop into mature fruits (Liu, 2006). Thus, mass pollination is necessary for the cross breeding of Chinese jujube. However, mass manual pollination of small flowers in Chinese jujube is very difficult

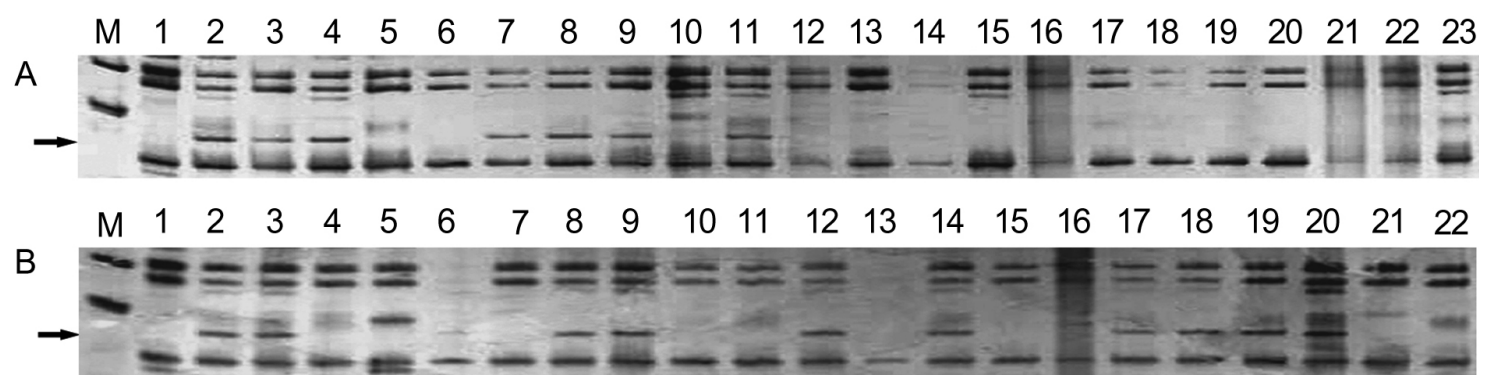

Fig. 3. One male-specific marker in JMS2 × 'Xing16'. Lane 1 (A and B), JMS2; lane 2 (A and B), 'Xing16'; lanes 3-23 (A), progeny No. 1-21; lanes 3-22 (B), progeny No. 22-41. Arrows indicate the male-specific marker.

Table 2. Plant regeneration and verification of hybrid progeny by AFLP.

\begin{tabular}{lccccc}
\hline $\begin{array}{l}\text { Cross } \\
\text { combination }\end{array}$ & $\begin{array}{c}\text { No. of } \\
\text { plantlets } \\
\text { from embryo }\end{array}$ & $\begin{array}{c}\text { No. of authentic } \\
\text { hybrids/ total } \\
\text { plantlets tested }\end{array}$ & $\begin{array}{c}\text { No. of plantlets } \\
\text { /No. of paternal } \\
\text { AFLP markers } \\
\text { shown in each }\end{array}$ & $\begin{array}{c}\text { Average No. of transmitted } \\
\text { paternal AFLP markers } \\
\text { /total paternal AFLP } \\
\text { markers used }\end{array}$ & $\begin{array}{c}\text { AFLP primers } \\
\text { (EcoRI/MseI) } \\
\text { used }\end{array}$ \\
\hline JMS2 $\times$ 'Xing16' & 105 & $41 / 41$ & $\begin{array}{c}2 / 5,9 / 4,16 / 3, \\
12 / 2,2 / 1\end{array}$ & $2.9 / 6$ & ATA/CAT, \\
AAC/CAT, \\
JMS2 $\times$ 'Xingguang'
\end{tabular}


and labor-consuming even with a male-sterile female parent. In this study, hybridization by manual pollination was also carried out from 2005 to 2007, in which one man only pollinated about 20 flowers per hour. We acquired only several fruits from about 3,000 flowers pollinated every year. Finally, we had to change pollination method and mass pollination was achieved by honeybee, which was an active pollinator of Chinese jujube (Ackerman, 1961). Obviously, the controlled pollination method using natural pollinator honeybee is more feasible and efficient than manual pollination in the cross breeding of Chinese jujube. In practice, selected male and female parents could be easily covered together by a cage by grafting them on the same tree or adjacent trees. Then, the honeybee-aided pollination could be easily accomplished.

Embryo culture has been utilized in the cross breeding of crops. The successful production of plants from the cultured embryos largely depends upon embryo age and medium composition (Raghavan, 2003; Ramming, 1990; Sharma et al., 1996; Zhu et al., 2013). Embryos of Chinese jujube abort mainly at hard stone stage of fruit. Embryos sampled before abortion are still young and their in vitro culture is very difficult (Liu and Qi, 2004; Wang et al., 2009). Although the plantlet regeneration rate from embryos increases at late fruit development stage, it is only about 30\% in JMS1 (Wang et al., 2009). The plant regeneration rate from embryos kept at $4^{\circ} \mathrm{C}$ for 40 days could reach $40 \%$ in culture of immature embryos obtained in JMS2 $\times$ 'Xingguang' and JMS2 $\times$ 'Xing16'. Compared with previous results, this study showed positive effects of the low temperature pretreatment on germination of immature embryo. However, the plant regeneration rate was still not high enough and should be increased with more measures. In several species of Prunus, immature embryos were usually kept at a low temperature $\left(0-4^{\circ} \mathrm{C}\right)$ more than 2 months to avoid the development of rosette type plants (Arbeloa et al., 2009; Burgos and Ledbetter, 1993; Kukharchyk and Kastrickaya, 2006). We noticed the formation of rosette-type structure without shoot tips from embryos which accounted for about $30 \%$ of the total cultured embryo in 2012. Finally, these embryos failed to germinate normally. Probably, 40 days at $4^{\circ} \mathrm{C}$ was not long enough for normal germination of immature embryos of Chinese jujube. It is necessary to explore more appropriate dormancy-breaking stimulus for Chinese jujube embryos.

Changes of the percentage of fruit with seed in different years revealed that hybridization was affected by not only cross combination but also other conditions such as relative humidity, sunlight, and tree condition. Although the changes also took place in JMS2 × 'Xingguang' and JMS2 × 'Xing16', progenies obtained from both combinations were much more than other cross combinations. Acquirement of high percentage of real hybrids demonstrated the compatibility between parents in the two combinations and the feasibility of hybridization and distant hybridization using JMS2 as a female parent. Sour jujube has some excellent traits such as resistance to saline and alkaline. Distant hybridization between Chinese jujube and sour jujube should play an important role in the improvement of Chinese jujube cultivars.

In conclusion, this study showed JMS2 was a new malesterile germplasm with stable male sterility. We have acquired its hybrids in the intra- and interspecific cross breeding via controlled hybridization. JMS2 is a promising female parent in the cross breeding of Chinese jujube. More cross combinations between JMS2 and other excellent genotypes of both Chinese jujube and sour jujube should be carried out urgently.

\section{Literature Cited}

Ackerman, W.L. 1961. Flowering, pollination, self-sterility, and seed development of Chinese jujube. Proc. Am. Soc. Hortic. Sci. 77:265-269.

Arbeloa, A., M. Daorden, E. García, P. Andreu, and J.A. Marín. 2009. In vitro culture of 'Myrobalan' (Prunus cerasifera Ehrh.) embryos. HortScience 44:1672-1674.

Asatryan A. and N. Tel-Zur. 2013. Pollen tube growth and selfincompatibility in three Ziziphus species (Rhamnaceae). FloraMorphology, Distribution, Functional Ecology of Plants 208:390-399.

Bai, R.X., J.Y. Peng, L. Li, Y. Zhang, and X.Y. Li. 2009. An improved protocol suitable for polymorphism studies in Chinese jujube (Ziziphus Jujuba Mill.). Acta Hortic. 840:97-106.

Burgos, L. and C.A. Ledbetter. 1993. Improved efficiency in apricot breeding: Effects of embryo development and nutrient media on in vitro germination and seedling establishment. Plant Cell Tiss. Org. Cult. 35:217-222.

Kukharchyk, N. and M. Kastrickaya. 2006. Embryo rescue techniques in Prunus L. breeding. J. Fruit Ornam. Plant Res. 14(Suppl. 1):129-135.

Li, Z.L. 1996. Tissue sections of plant. Peking Univ. Press, Beijing, China.

Liu, M.J. 2006. Chinese jujube: Botany and horticulture. Hortic. Rev. 32:229-298.

Liu, M.J. and M. Wang. 2009. Germplasm resources of Chinese jujube. China For. Publ. House, Beijing, China.

Liu, M.J. and Y.F. Qi. 2004. Embryo rescue of Chinese jujube (Ziziphus Jujuba Mill.). Acta Hortic. 663:479-482.

Liu, M.J., J.Y. Zhou, J. Zhao, J.R. Wang, P. Liu, L. Dai, and Y.X. Wang. 2006. An excellent new cultivar of Chinese jujube with 
high resistance to jujube witches broom disease 'Xingguang'. Acta Hortic. Sin. 33:687.

Liu, P., L. Dai, M.J. Liu, H.E. Jiang, Z.H. Zhao, and J.R. Wang. 2012. 'Chenguang', a new tetraploid Chinese jujube cultivar. Fruits 67:293-296.

Lu, J.Y., Y.M. Mao, L.Y. Shen, S.Q. Peng, and M. Liu. 2005. Application of AFLP markers for identification of hybrids from open pollinated Dongzao (Zizyphus jujuba Mill.) progenies. Acta Hortic. Sin. 32:680-683.

Lyrene, P.M. 1983. Flowering and fruiting of Chinese jujubes in Florida. HortScience 18:208-209.

Pooler, M.R., L.G. Riedel, S.E. Bentz, and A.M. Townsend. 2002. Molecular markers used to verify interspecific hybridization between hemlock (Tsuga) species. J. Am. Soc. Hortic. Sci. 127:623-627.

Raghavan, V. 2003. One hundred years of zygotic embryos culture investigations. In Vitro Cell Devel. 39:437-442.

Ramming, D.W. 1990. The use of embryo culture in fruit breeding. HortScience 25:393-398.

Sharma, D.R., R. Kaur, and K. Kumar. 1996. Embryo rescue in plants: A review. Euphytica 89:325-337.

Wang, J.R., L. Liu, M.J. Liu, and J.Y. Zhou. 2007. Acquirement of a new male sterile germplasm of Chinese jujube. Front. Agr. China 1:72-75.

Wang, J.R., M. Zhang, and M.J. Liu. 2009. Plant regeneration from immature embryo in Chinese jujube (Ziziphus jujuba
Mill.). Acta Hortic. 840:265-272.

Wang, J.R., M.J. Liu, and L. Dai. 2003. Difference of cultivars in Chinese jujube tissue culture and micropropagation of Lajiaozao. J. Agr. Univ. Hebei 26:59-61.

Whittemore, A.T. and A.M. Townsend. 2007. Hybridization and self-compatibility in Celtis: AFLP analysis of controlled crosses. J. Am. Soc. Hortic. Sci. 132:368-373.

Yan, C., P. Liu, M.J. Liu, J.R. Wang, D.C. Kong, and D.K. Li. 2010. The influencing factors of the fruiting characteristics of Ziziphus jujuba. Sci. Silvae Sinicae 46:78-86.

Yan, C., P. Liu, M.J. Liu, J.R. Wang, L. Dai, D.K. Li, and D.C. Kong. 2009. Characteristics of fruiting and fertility of different cultivars of Ziziphus jujuba Mill. J. Plant Genet. Resour. 10:121-125.

Yao, S.R. 2013. Past, present, and future of jujubes: Chinese dates in the United States. HortScience 48:672-680.

Zhang, S.L., S.M. Cao, and H.Q. Wu. 2003. Self-incompatibility genotypes of fruit trees and their identification methods. J. Fruit Sci. 20:358-363.

Zhang, W.C. 2001. Methods in fruit studies. China Agr. Press, Beijing, China.

Zhu, W.Y., J.F. Jiang, S.M. Chen, L. Wang, L.L. Xu, H.B. Wang, P.L. Li, Z.Y. Guan, and F.D. Chen. 2013. Intergeneric hybrid between Chrysanthemum $\times$ morifolium and Artemisia japonica achieved via embryo rescue shows salt tolerance. Euphytica 191:109-119. 